\title{
COOLING OF DENSE STARS
}

\author{
SACHIKO TSURUTA* \\ NASA, Goddard Space Flight Center, Greenbelt, Md., U.S.A.
}

\begin{abstract}
Cooling rates are first calculated for neutron stars of about $1 M_{\odot}$ and $10 \mathrm{~km}$ radius, with magnetic fields from zero to about $10^{14} \mathrm{G}$, for two extreme cases of maximum and no superfluidity. The results show that most pulsars are so cold that thermal ionization of surface atoms would be negligible. Next, nucleon superfluidity and crystallization of heavy nuclei are treated more quantitatively, and more realistic hadron star models are chosen. Cooling rates are thus calculated for a stable hyperon star near the maximum mass limit, a medium weight neutron star, and a light neutron star with neutron-rich heavy nuclei near the minimum mass limit. Results show that cooling rates are a sensitive function of density. The lightest star is cooler than others in earlier stages but the trend is reversed later. The medium weight star is generally the coldest of all in lower temperature regions where the effect of superfluidity becomes significant. However, if a heavy star contains pions, its cooling will be even faster. The Crab pulsar and Vela pulsar, expected to be the two youngest, can be as hot as $(2 \sim 4) \times 10^{6} \mathrm{~K}$ (on the surface), comparable with the results obtained from internal frictional heating by Greenstein, if they are medium weight to heavy hadron stars. However, older pulsars are cold. In fact, at about a few million years, the age of average radio pulsars, the surface temperature becomes only several hundred to several thousand degrees. Thus, the earlier conclusions about cold pulsars are still valid. Cooling of a massive white dwarf star is also shown.
\end{abstract}

\section{Introduction}

In recent years considerable progress has been made in the studies of cold dense matter. In the present paper, we wish to report some recent work on thermal properties of dense stars.

Some years ago when the first few of the galactic X-ray sources were discovered,it was suggested that these $\mathrm{X}$-ray sources might be neutron stars. The detailed account was reviewed, for instance, in the paper by Tsuruta and Cameron (1966a). The conclusion was that the cooling rate alone could not exclude the possibility of detecting neutron stars as X-ray emitters, but that the observed spectra of X-rays from these sources were inconsistent with those of black-body radiation expected from the surface of neutron stars. This and other considerations led us to doubt the prospect of ever observing neutron stars. The discovery of pulsars several years ago, however, changed the whole picture. Now it is generally believed that pulsars are rotating, magnetic neutron stars (Gold, 1968). Moreover, theoretical considerations and some observational evidence (such as the speed-ups of the Crab and Vela pulsars) suggest the presence of superfluids in neutron stars (Ginzburg, 1971; Borner and Cohen, 1972; and the paper by Pines in this volume). In the earlier cooling calculations the effect of magnetic fields and superfluidity was not taken into account. In our recent work, therefore, emphasis was placed on the effect of these new factors, which were expected to reduce cooling rates significantly. The new outcome may prove valuable for the understanding of pulsar and X-ray star problems.

* National Academy of Science-NRC Senior Research Associate at NASA, Goddard Space Flight Center. 
In Section 2 the basic equations are introduced. In Section 3.1 we summarize the general effect of strong magnetic fields and superfluidity on neutron star cooling - a joint work of Ruderman, Canuto, Lodenquai and myself, published in Tsuruta et al. (1972). In recent months I have tried to treat the problem more quantitatively using realistic hadron star models and other newly available information. The results are reported in Section 3.2. Cooling of white dwarfs is discussed in Section 3.3.

\section{Basic Equations}

The cooling time $t$ is defined by

$$
t=\int_{U_{0}}^{U_{t}} \frac{\mathrm{d} U}{\bar{L}(U)},
$$

where $U_{0}$ and $U_{t}$ are the energies of the star at $t=0$ and at time $t$, respectively, and $\mathrm{L}(U)$ is the average luminosity over the interval $\mathrm{d} U$. The total energy is mainly a function of the internal temperature of the star $T_{i}$ and is expressed as

$$
U=U\left(T_{i}\right)=\sum_{k} U_{k}^{D}+U_{\mathrm{ion}}^{N} \quad(\text { with } k=\mathrm{n}, \mathrm{p}, \mathrm{e}, \mathrm{m}, \mathrm{h}) .
$$

The sum is over the thermal tails of degenerate particles $k$ and $U_{\text {ion }}^{N}$ is the energy of the non-degenerate heavy ions. Here $k$ labels neutrons (n), protons (p), electrons (e), mesons (m), and hyperons (h).

The total energy loss rate $L$ is

$$
\begin{aligned}
L & =L_{\gamma}+L_{v}, \\
L_{\gamma} & =L_{\gamma}\left(T_{\mathrm{e}}\right)=4 \pi \sigma R^{2} T_{\mathrm{e}}^{4}, \\
L_{v} & =L_{v}\left(T_{\mathrm{i}}\right)=L_{v}^{u}+L_{v}^{B}+L_{v}^{p 1}+L_{v} \text { (others), }
\end{aligned}
$$

$L_{\gamma}$ is the photon luminosity and $L_{v}$ is the neutrino luminosity. The superscripts $u, B$, and $p l$ stand for the neutrino URCA, bremsstrahlung, and plasmon processes, respectively. $L_{v}$ (others) accounts for photoneutrino, pair annihilation and neutrino synchrotron losses. In the earlier stages of cooling, when temperatures are high, neutrino emission dominates, while later, at lower temperatures, photon emission is more significant. In the absence of magnetic fields and superfluidity, the URCA process is dominant among neutrino processes though the plasmon process competes with it at higher temperatures and bremsstrahlung at lower temperatures. At extremely high temperatures $\left(T>10^{10} \mathrm{~K}\right)$ pair annihilation becomes significant, but contributions by other process are relatively minor.

The total energy. and neutrino luminosity depend on the internal temperature, whereas the photon luminosity is a function of surface temperature (and radius). Thus, the first step is to find the relation between $T_{i}$ and $T_{e}$. For this purpose the energy transfer equation and hydrostatic equations are integrated over the outer layers of the star from the surface to the point where the temperature gradient disappears. Recent 
studies suggest that a neutron star has a distinct boundary where $\varrho_{\mathrm{s}} \simeq 10^{4} \mathrm{~g} \mathrm{~cm}^{-3}$ and a sharp, discontinuous density drop thereafter (see, for example Ruderman's paper in this volume). Therefore, this integration was performed both from the photosphere and from the boundary with $\varrho_{\mathrm{s}} \simeq 10^{4} \mathrm{~g} \mathrm{~cm}^{-3}$. For magnetic neutron stars with $H \gtrsim 10^{12} \mathrm{G}$ both methods led to similar results. Convection is very unlikely in neutron stars (Tsuruta, 1964), so that energy transfer will be governed by radiation. The basic equations are therefore expressed as (Tsuruta and Cameron, 1966a)

$$
\begin{aligned}
\frac{\mathrm{d} T}{\mathrm{~d} r} & =-\frac{1}{4 \pi r^{2}} \frac{3}{4 a c} \frac{\kappa \varrho}{T^{3}} L(r), \\
\frac{\mathrm{d} P}{\mathrm{~d} r} & =-\frac{G\left(P / c^{2}+\varrho\right)\left(4 \pi r^{3} P / c^{2}+m\right)}{r\left(r-2 m G / c^{2}\right)} \\
\frac{\mathrm{d} m}{\mathrm{~d} r} & =4 \pi \varrho r^{2},
\end{aligned}
$$

along with the equation of state

$$
P=P(\varrho, T),
$$

which becomes degenerate at higher densities $\left(\varrho \gtrsim 10^{4}\right.$ to $10^{6} \mathrm{~g} \mathrm{~cm}^{-3}$ depending on temperature). The opacity which appears in the transfer equation is expressed as

$$
\frac{1}{\kappa}=\frac{1}{\kappa_{\mathrm{R}}}+\frac{1}{\kappa_{\mathrm{C}}},
$$

where $\kappa_{\mathrm{R}}$ and $\kappa_{\mathrm{C}}$ are the radiative and conductive opacities, respectively.

\section{Results and Conclusions}

\subsection{GENERAL EFFECTS OF MAGNETIC FIELDS AND SUPERFLUIDITY ON COOLING}

For this purpose we shall use the ' $V_{\gamma}$ (II)' medium mass neutron star model of Tsuruta and Cameron (1966b) (see also Hartle and Thorne, 1968). The equation of state for this model is derived partially from the $V_{\gamma}$ potential of Levinger and Simmons. Properties of the model are: $M=1.07 M_{\odot}, R=12.33 \mathrm{~km}$, and $\varrho_{\mathrm{c}}$ (central density)= $=7.39 \times 10^{14} \mathrm{~g} \mathrm{~cm}^{-3}$.

The major effect of strong magnetic fields is to drastically reduce photon opacities in certain spatial directions. It also reduces significantly the URCA neutrino luminosity. The effect of the presence of superfluidity is mainly to reduce the total energy of superfluid particles at lower temperatures. It also suppresses the URCA luminosity.

First, consider the effect of magnetic fields on opacity. We have found that the following approximation is valid for the radiative opacity in superstrong magnetic fields with $H \gtrsim 10^{12} \mathrm{G}$ (see Tsuruta et al., 1972; Lodenquai et al., 1974; Canuto et al., 1971; Canuto, 1970):

$$
\sigma_{\omega}(H)=\left(\frac{\omega}{\omega_{\mathrm{H}}}\right)^{2} \sigma_{\omega}(0), \text { for } \omega \ll \omega_{\mathrm{H}},
$$




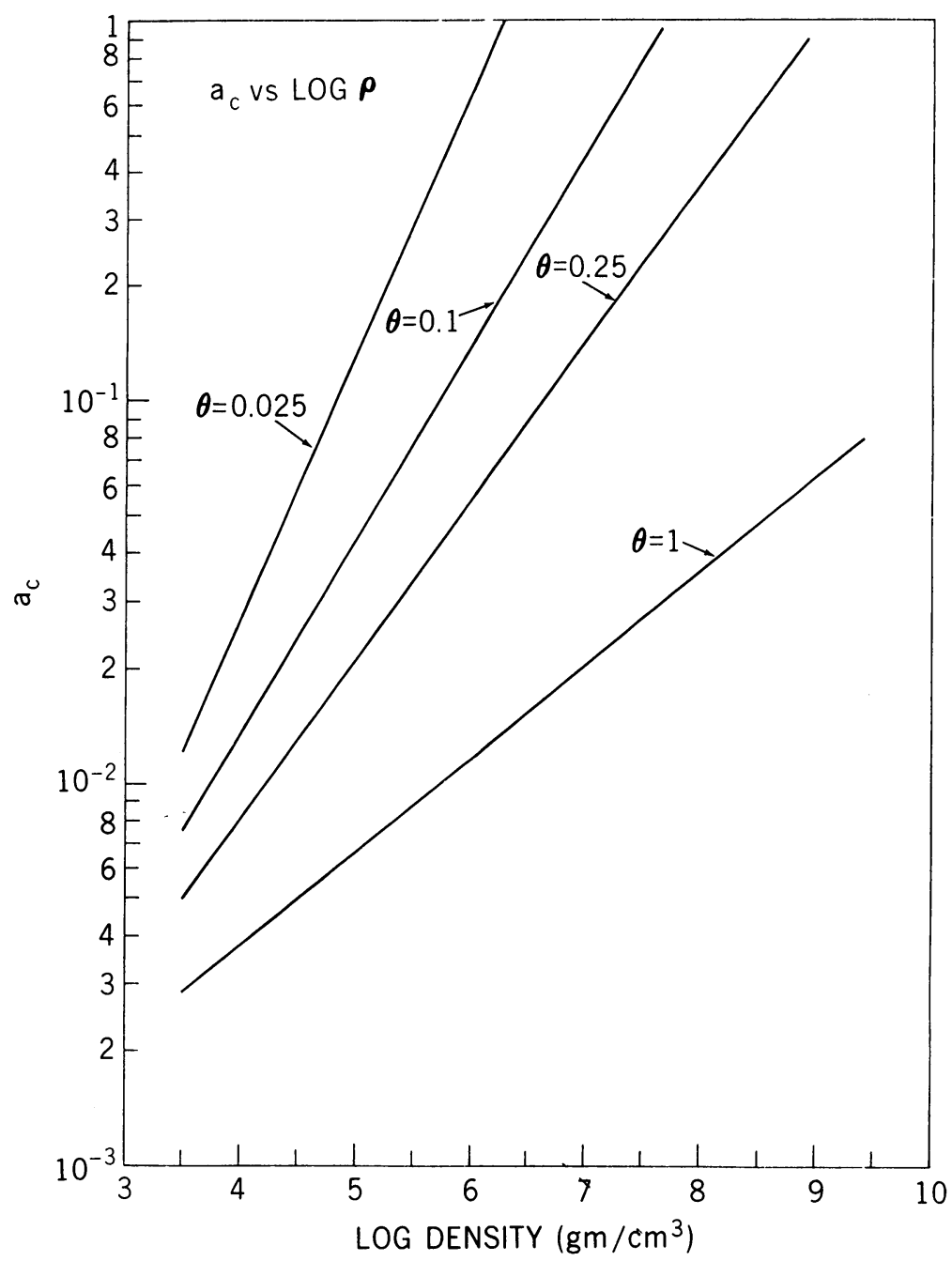

Fig. 1. The factor $a_{\mathrm{c}}$ as a function of density. The $a_{\mathrm{c}}$ is the ratio of conductive opacity with magnetic fields to that without magnetic fields, $\kappa_{\mathrm{c}}(H) / \kappa_{\mathrm{c}}(0) . \theta=H / H_{q}$, and $H_{q}=4.41 \times 10^{13} \mathrm{G}$.

where $\omega$ is the radiation frequency, $\omega_{\mathrm{H}}=e H / m_{\mathrm{e}} c$ is the electron cyclotron frequency, and $\sigma_{\omega}(H)$ and $\sigma_{\omega}(0)$ are the photon cross sections with and without a magnetic field, respectively. The Rosseland mean was used to take account of the frequency dependence. The radiative opacity, thus obtained, can be expressed as

$$
\kappa_{\mathrm{R}}(H)=a_{\mathrm{R}} \kappa_{\mathrm{R}}(0) ; \text { with } a_{\mathrm{R}} \propto\left(\frac{T}{H}\right)^{2} \leqslant 1
$$

The conductive opacity is similarly expressed as

$$
\kappa_{\mathrm{c}}(H)=a_{\mathrm{c}} \kappa_{\mathrm{c}}(0), \quad a_{\mathrm{c}} \leqslant 1 .
$$




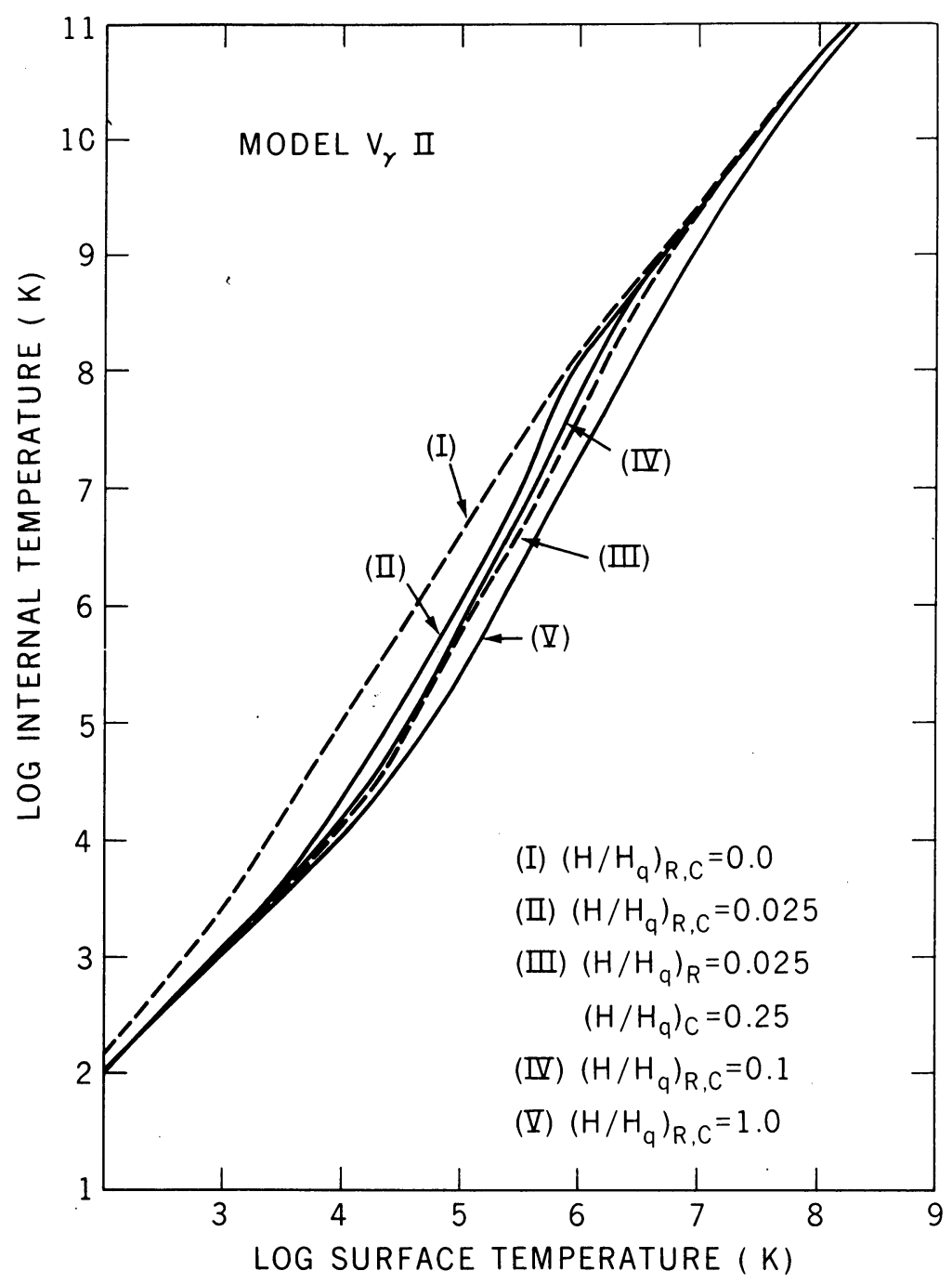

Fig. 2. Internal temperature, as a function of surface temperature at different magnetic field strengths, for Model $V_{\gamma} I I$ as described in Section 3.1. Curves (I), (II), (IV) and (V) stand for a uniform magnetic field of strengths $\left(H / H_{q}\right)=0,0.025,0.1$ and 1 , respectively. In the curve (III), the surface field strength is the same as in the curve (II), but the internal field strength is increased by a factor of 10 .

The correction factor $a_{c}$ is generally a complicated function of the magnetic field, density and temperature. In the inner degenerate layers where the conductive opacity plays the dominant role, the temperature dependence drops out and the factor $a_{\mathrm{c}}$ decreases with increasing magnetic field strengths and decreasing density. In Figure 1 the density dependence of the factor $a_{\mathrm{c}}$ is shown for different magnetic field values in the regions of interest. Here, $\theta=H / H_{q}$, with $H_{q}=m_{\mathrm{e}}^{2} c^{3} / \hbar e=4.41 \times 10^{13} \mathrm{G}$. (Similar calculations were made by Canuto and Chiu (1969) for $\theta=1$ and 0.1 in the vicinity of 


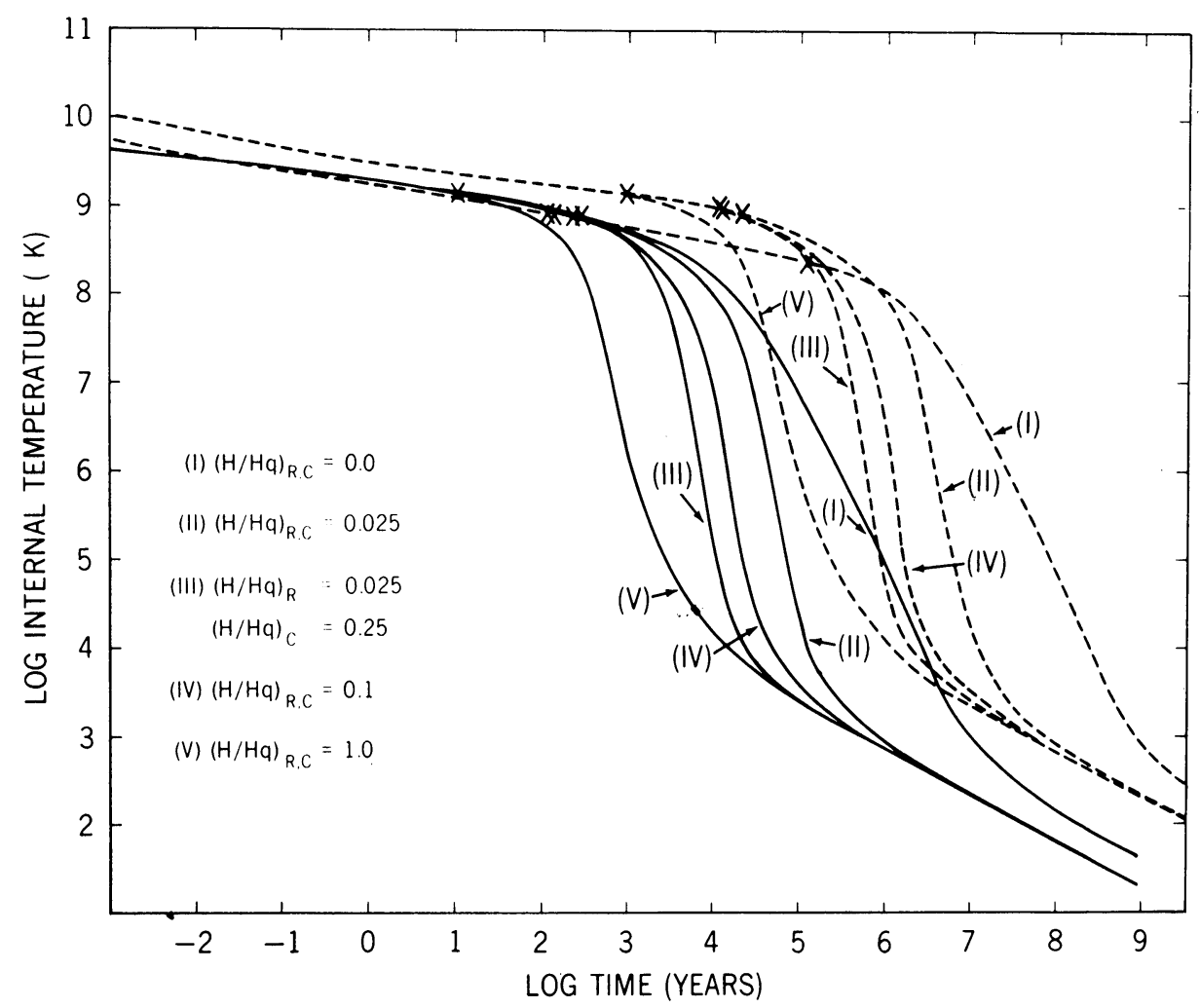

Fig. 3. Internal temperature as a function of time for Model $V_{\gamma} \mathrm{II}$ at different magnetic field strengths. Notation is the same as in Figure 2. The points where the major cooling mechanism shifts from neutrino emission to photon emission are indicated by the crosses. The solid curves and dashed curves represent two extreme cases of maximum and no superfluidity, respectively, as explained in the text.

$\varrho \simeq 10^{6} \mathrm{~g} \mathrm{~cm}^{-3}$.) We have applied these correction factors to the zero field opacities previously obtained by Tsuruta and Cameron (1966a). It may be noted that electrons become relativistic for densities $\varrho \gtrsim 10^{6} \mathrm{~g} \mathrm{~cm}^{-3}$. In these high density regions, therefore, we used the formula for conductive opacity (which applies to both relativistic and non-relativistic cases), given by Schatzman (1958). (In the earlier work where the Las Alamos opacity code was used for zero field opacities, the relativity effect was not included. I thank Professor Hayashi for pointing out this to me.)

In Figure 2, the relation between $T_{\mathrm{i}}$ and $T_{\mathrm{e}}$ thus obtained is shown for varying strengths of magnetic fields. In the curve (I), $H=0$. The curve (II) corresponds to a star of constant field strength of about $10^{12} \mathrm{G}$. In the curve (III), the surface field is the same as in (II) but the internal field is increased by a factor of 10 . We note that the difference between the internal temperature and surface temperature decreases with increasing $H$ and decreasing $T$. When $H \gtrsim 10^{12} \mathrm{G}$, this difference almost vanishes for $T \$ 1000 \mathrm{~K}$.

In order to study the effect of superfluidity, the following two extreme cases are 


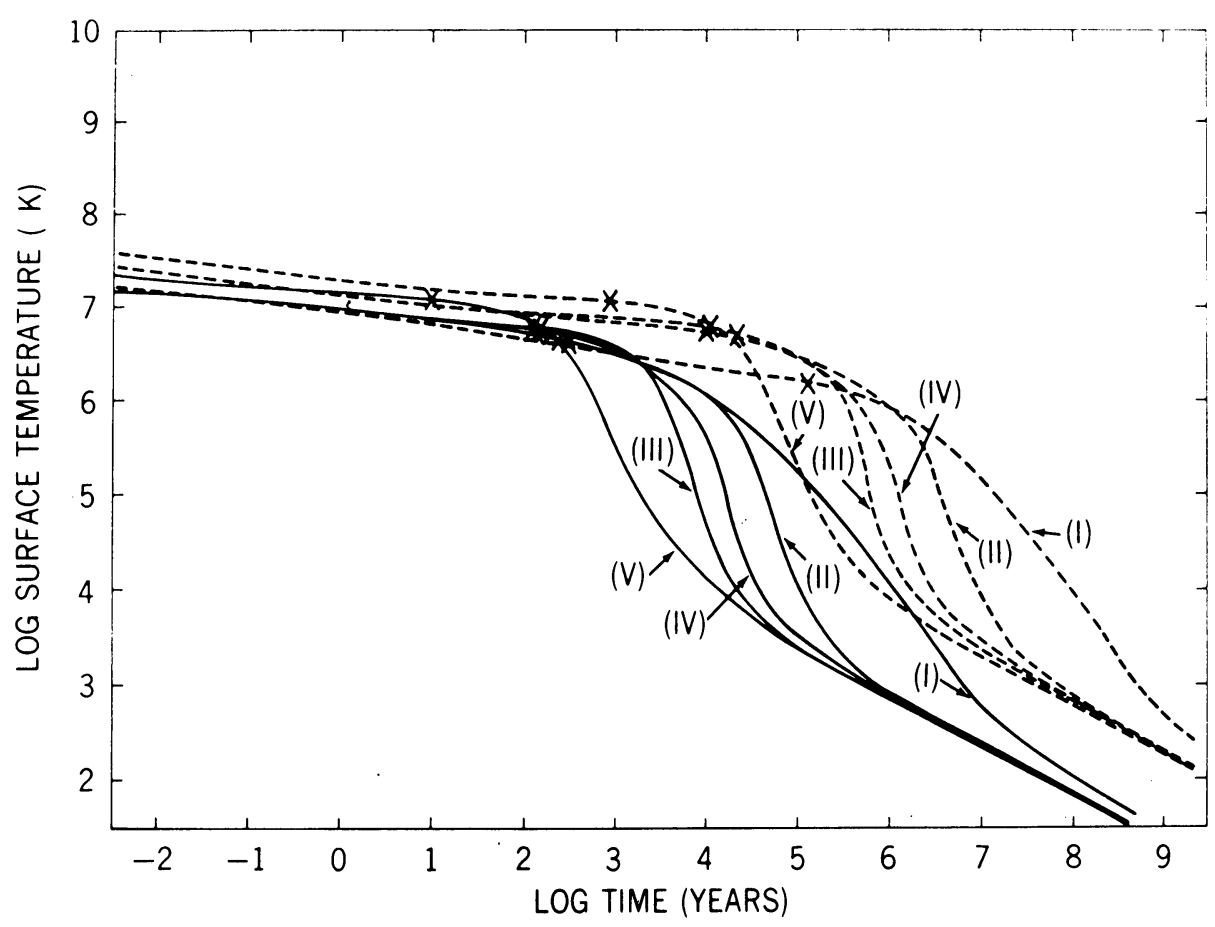

Fig. 4. Surface temperature as a function of time (cooling curves) for Model $V_{\gamma} \mathrm{II}$ at different magnetic field strengths. Notation is the same as in Figure 3.

studied. In Case (S) (maximum superfluidity), the energy of superfluid particles (neutrons and protons) is set equal to zero, and thus degenerate electrons are the main contributors to the total energy. In Case (N) (normal state), superfluidity is completely neglected, and thus degenerate neutrons are the main contributors to the total energy. These effects on the URCA luminosity are also maximized. Thus, we set $L_{v}^{u}=0$ for all models in Case (S) and for $H \geqslant 10^{12} \mathrm{G}$ in Case (N). The results are shown in Figures 3 and 4 , where internal temperatures and surface temperatures, respectively, are plotted as functions of time for different magnetic field strengths. The solid curves stand for Case (S) and the dashed curves represent Case $(\mathrm{N})$.

We note that the effect of both strong magnetic fields and superfluidity are important for neutrons stars older than about $100 \mathrm{yr}$. For instance, at $t=1000 \mathrm{yr}$ (which corresponds to the approximate age of the Crab pulsar NP 0532) the surface temperature is anywhere between $\sim 10^{5} \mathrm{~K}$ and a few times $10^{7} \mathrm{~K}$. For the Vela pulsar (with $t \simeq 10^{4}$ years) it is between $10^{4} \mathrm{~K}$ and $10^{7} \mathrm{~K}$. However, for typical older pulsars of a few million years (so far observed only as radio pulsars), the star seems very cold (with the surface temperature anywhere between about $10^{2} \mathrm{~K}$ and $10^{5} \mathrm{~K}$ ).

\subsection{Application to SPECIFIC MÓDELS}

In view of the large discrepancy found in the last section between the two extreme 


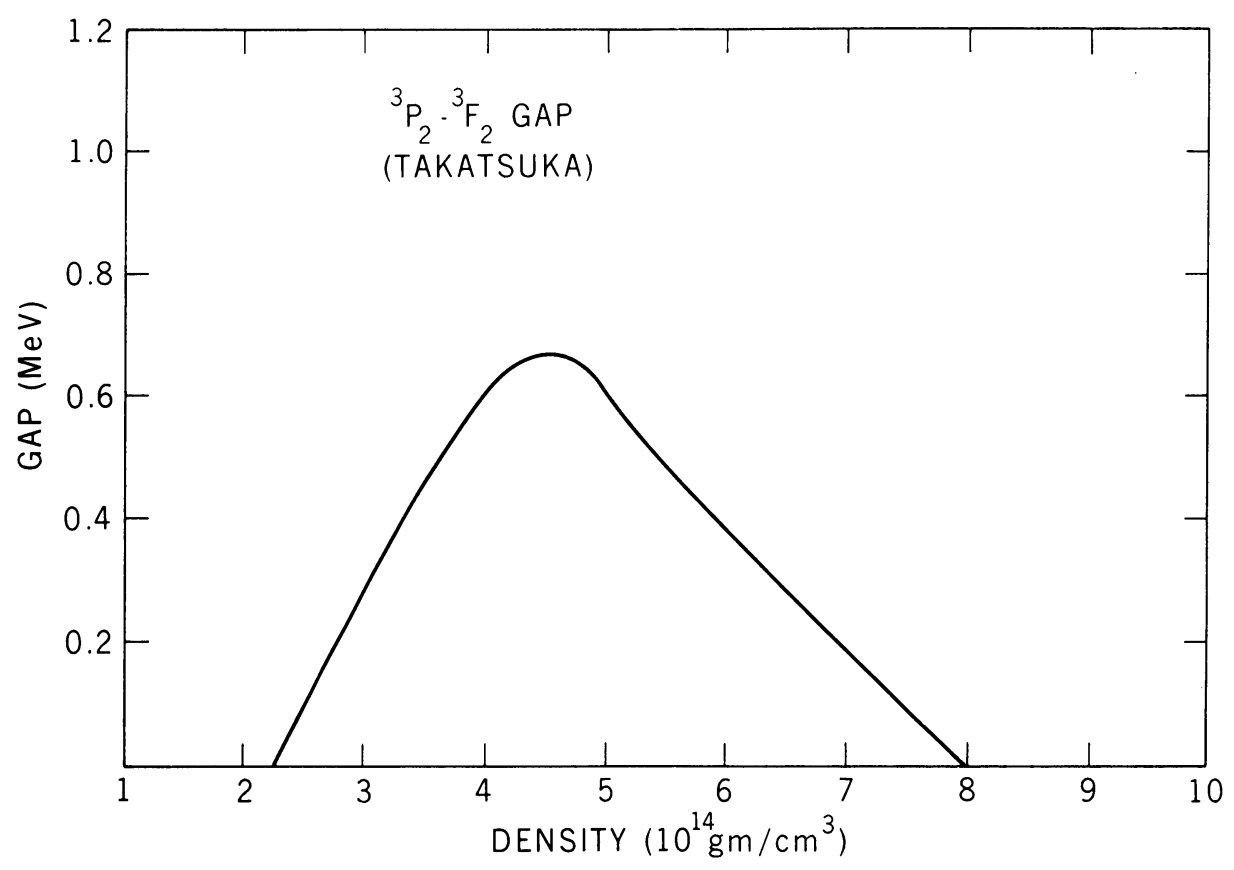

Fig. 5. The density dependence of $\Delta\left({ }^{3} P_{2}\right)$ calculated by Takatsuka (1972) including the ${ }^{3} P_{2}-{ }^{3} F_{2}$ tensor coupling.

cases (of maximum and no superfluidity), it might be worthwhile to treat more exactly the effect of superfluidity. Fortunately, the large uncertainty concerning the superfluid energy gap was greatly reduced according to the latest report from the Kyoto group (Takatsuka, 1972; Tamagaki, 1972). There, the ${ }^{1} S_{0}$-gap and the ${ }^{3} P_{2}$-gap, $\Delta\left({ }^{1} S_{0}\right)$ and $\Delta\left({ }^{3} P_{2}\right)$, are calculated as a function of density by making use of realistic values of the effective mass, $m^{*}(\varrho)$. Due to a slight decrease of the value $m^{*}$, the gap $\Delta\left({ }^{1} S_{0}\right)$ is somewhat reduced but otherwise its general behavior is similar to previous results. There are, however, significant changes in the behavior of the gap $\Delta\left({ }^{3} P_{2}\right)$. Taking into account the attractive effect of the ${ }^{3} \mathrm{P}_{2}-{ }^{3} \mathrm{~F}_{2}$ coupling due to the two-nucleon tensor force, Takatsuka (1972) shows that the gap $\Delta\left({ }^{3} P_{2}\right)$ behaves as shown in Figure 5. (If the ${ }^{3} P_{2}{ }^{3} F_{2}$ coupling is neglected, the ${ }^{3} P_{2}$-gap becomes negligibly small for realistic values of $m^{*}$.) The conclusion is; (i) the ${ }^{1} S_{0}$-gap (with maximum $\Delta \simeq 2.5 \mathrm{MeV}$ ) exists in the density region $\varrho \simeq\left(10^{11} \sim 1.5 \times 10^{14}\right) \mathrm{g} \mathrm{cm}^{-3}$ and, (ii) the ${ }^{3} P_{2}$-gap (with maximum $\Delta \simeq 0.65 \mathrm{MeV}$ ) exists in the range $\varrho \simeq(2 \sim 8) \times 10^{14} \mathrm{~g} \mathrm{~cm}^{-3}$.

It seems clear from the above that the appearance of superfluidity depends critically on the stellar density. If the density is as high as $\sim 10^{16} . \mathrm{g} \mathrm{cm}^{-3}$ for stars near the maximum mass limit (as some neutron star models indicate), the effect of superfluidity will be small. (These heavy stars may possibly contain high concentrations of hyperons and may better be called 'hyperon' or 'hadron stars.') On the other hand, lighter stars near the minimum mass limit may have quite different internal structures; namely the 
presence of heavy nuclei in the whole interior (Baym et al., 1971b). Therefore, three typical models are chosen for the present investigation. These are: Model (I) a heavy hyperon star near the maximum mass limit, Model (II) a medium weight neutron star, and Model (III) a light neutron star near the minimum mass limit whose central core contains heavy nuclei. Their characteristics are summarized in Table I. In these models the different density ranges are treated in the following manner:

TABLE I

Characteristics of the three models of hadron stars chosen in Section 3.2

\begin{tabular}{|c|c|c|c|c|}
\hline \multirow[t]{2}{*}{ Model } & $\begin{array}{l}\text { Central } \\
\text { density }\end{array}$ & Mass & Radius & \\
\hline & $Q_{\mathrm{c}}\left(\mathrm{g} \mathrm{cm}^{-3}\right)$ & $M / M_{\odot}$ & $R(\mathrm{~km})$ & \\
\hline (I) & $6.0 \times 10^{15}$ & 1.413 & 7.10 & Heavy \\
\hline (II) & $8.0 \times 10^{14}$ & 0.476 & 10.9 & Medium \\
\hline (III) & $1.1 \times 10^{14}$ & 0.105 & 76.7 & Light \\
\hline
\end{tabular}

Note: $Q_{\mathrm{c}}$ is the central (energy) density in cgs units, $M / M_{\odot}$ is the mass in solar mass units, and $R$ is the stellar radius in $\mathrm{km}$. Model (I) is a heavy hyperon star, Model (II) is a medium weight neutron star, and Model (III) is a light neutron star with neutron-rich heavy nuclei.

For the outermost layers, where the density $\varrho<\varrho_{1}=3 \times 10^{11} \mathrm{~g} \mathrm{~cm}^{-3}$, the temperature dependence of composition cannot be neglected for our present purposes. This is mainly because the neutron abundance is significant at higher temperatures, while neutrons disappear in this region at zero temperatures (Salpeter, 1961; Wheeler, 1966). In fact, it was found that the matter becomes predominantly composed of neutrons (with small percentages of $\alpha$-particles, protons and electrons) for $T \gtrsim 2 \times 10^{10} \mathrm{~K}$. At about $4 \times 10^{9} \mathrm{~K}$, where the composition freezes, there are still significant numbers of neutrons present. Also on the high density side $\left(\varrho \gtrsim 10^{11} \mathrm{~g} \mathrm{~cm}^{-3}\right)$ heavy nuclei are crystallized before the composition freezes. Therefore, we used the results of recent calculations of equilibrium compositions at finite temperatures $\left(10^{9} \sim 5 \times 10^{10} \mathrm{~K}\right)$ by Tsuruta et al. (1973).

In the subnuclear density region, where $\varrho_{1} \lesssim \varrho<\varrho_{2} \equiv \sim 2 \times 10^{14} \mathrm{~g} \mathrm{~cm}^{-3}$, the matter consists of neutron-rich heavy nuclei $(A, Z)$, neutrons and electrons. For the value $Z$, we used the recent work by Ravenhall et al. (1972). They show that in this region the value $Z$ is a finite and slowly varying function of density. The ratio $A / Z$ at a given density is more insensitive to different models, and we used the values listed in Baym et al. (1971a). The relative abundance of each component was then calculated using standard methods (see the above references).

In the nuclear region, where $\varrho_{2} \lesssim \varrho<\varrho_{3}=\sim 8 \times 10^{14} \mathrm{~g} \mathrm{~cm}^{-3}$, we assumed the OPEG type potential for nucleon interactions. This is a Gaussian type soft core potential with one pion exchange constructed by Tamagaki (1968). The relative abundances 
(neutrons, protons and electrons) was found by a standard method (see, for instance, Baym et al., 1971a).

In the ultradense region, where $\varrho \gtrsim \varrho_{3}$ the presence of hyperons and muons was assumed. Specifically, the hyperonic composition of model (C) by Pandharipande (1971) was adopted. It may be noted that the appearance of hyperons depends critically on the type of strong interactions assumed among baryons. For instance, it was reported (Moszkowski, 1972) that at above 6 times nuclear density the most stable configuration is still a neutron gas in certain cases. This is for a certain baryon-baryon interaction which he calls the 'Modified Delta Interaction.' However, for a Reid soft

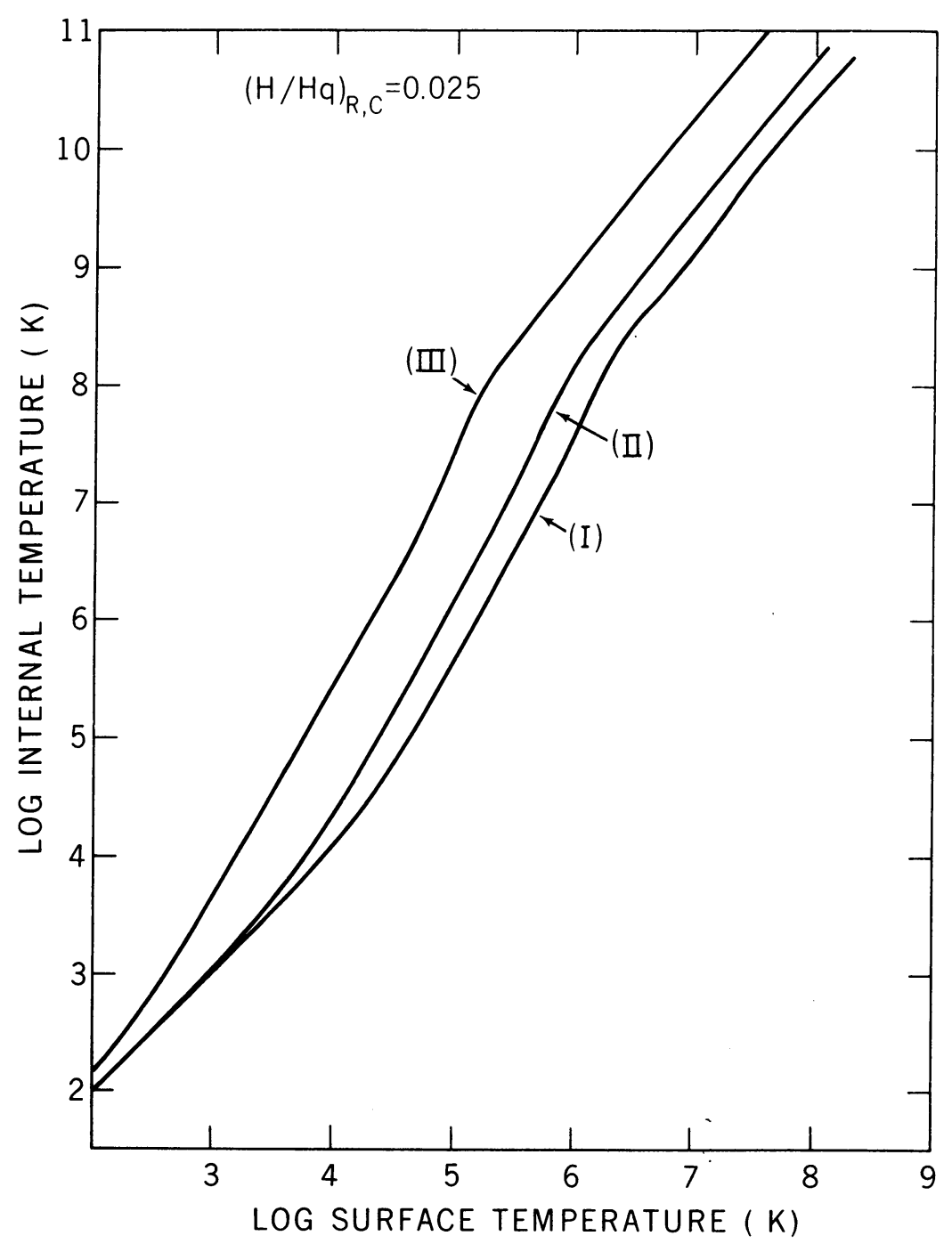

Fig. 6. Internal temperature as a function of surface temperature for the three dense star models (I), (II) and (III) chosen in Section 3.2. A uniform magnetic field of strength $\left(H / H_{q}\right)=0.025$ is used. 
core baryon-baryon interaction with a $G$-matrix calculated by Sawada and Wong (private communication), there appear significant fractions of hyperons at supernuclear densities (Moszkowski, private communication). (However, both interactions lead to very similar equations of state for pure neutron matter.) In any case, the results of our cooling calculations do not change significantly if our heaviest model is a neutron star instead of a hyperon star.

Using (4) to (8), the relation between internal temperatures and surface temperatures was obtained for the models (I), (II) and (III) described above. The results are shown in Figure 6. In this connection, we may note the angular dependence of the magnetic reduction of opacity. This problem was taken into account by assuming a dipole field and by the estimate that the reduction as expressed by (6) applies in directions within about $10 \%$ along both magnetic poles. This approximation is thought to be sufficient in view of greater uncertainties involved in the derivation of (7) and (8).

In the final cooling calculations, the energy and the URCA rates of superfluid nucleons were treated more accurately. We also included the crystallization of heavy ions. Thus, as the energy of degenerate particles $k$ in (2), we used the following formulae:

$$
\begin{aligned}
U_{k}^{D} & =\int C_{k}^{D} \mathrm{~d} T \\
C_{k}^{D} & =\int\left(C_{0}\right)_{k}^{D} Y_{\mathrm{s}} n_{k} 4 \pi r^{2} \mathrm{~d} r \\
\left(C_{0}\right)_{k}^{D} & =\left(\frac{\pi^{2} k_{\mathrm{B}}^{2}}{m_{k} c^{2}} \frac{\left(x_{k}^{2}+1\right)^{1 / 2}}{x_{k}^{2}}\right) T, \\
x_{k} & =P_{k}^{F} /\left(m_{k} c\right), \\
Y_{\mathrm{s}} & =\frac{8.5 T_{\mathrm{c}}}{T} \exp \left(-1.44 \frac{T_{\mathrm{c}}}{T}\right), \text { for } T \ll T_{\mathrm{c}}, \\
k_{\mathrm{B}} T_{\mathrm{c}} & =0.57 \times \Delta\left({ }^{3} P_{2}\right) / \sqrt{2} \Gamma_{0} \quad\left(\text { with } \ln \Gamma_{0}=1.22\right)
\end{aligned}
$$

or

$$
\dot{k}_{\mathrm{B}} T_{\mathrm{c}}=0.57 \times \Delta\left({ }^{1} S_{0}\right) .
$$

$Y_{\mathrm{s}}$ is a superfluid correction factor which becomes unity in the absence of superfluidity. The intermediate region between $T=T_{\mathrm{c}}$ and $T \ll T_{\mathrm{c}}$ was interpolated. The thermal energy of heavy ions is expressed as

where

$$
U_{\mathrm{ion}}^{N}=\left[\int c_{v} \dot{n}_{\mathrm{ion}} 4 \pi r^{2} \mathrm{~d} r\right] T,
$$

$$
\begin{aligned}
& c_{v}=a_{i} k_{\mathrm{B}} \mathscr{D}\left(\frac{\theta}{T}\right) \\
& a_{i}=\frac{3}{2} \text { for } T \geqslant T_{\mathrm{g}}
\end{aligned}
$$

and

$$
a_{i}=3 \text { for } T \leqslant T_{\mathrm{m}} \text {. }
$$


$T_{\mathrm{g}}$ is the temperature above which ions become a gas and $T_{\mathrm{m}}$ is the melting temperature. In the intermediate region between $T_{\mathrm{g}}$ and $T_{\mathrm{m}}$, the ions form a liquid and the constant $a_{i}$ takes on values intermediate between 1.5 and 3. The expression $\mathscr{D}(\theta / T)$ is the Debye function for crystals, which approaches zero as $(T / \theta)^{3}$ for $T \ll T_{\mathrm{m}}$ (Landau and Lifshitz, 1958). The values $T_{\mathrm{m}}$ and $T_{\mathrm{g}}$ were estimated from the work of Van Horn (1968) and Mestel and Ruderman (1967). In the above equations, $k_{\mathrm{B}}$ is Boltzmann's constant, and $n_{k}$ and $n_{\text {ion }}$ are the number densities of particles $k$ and ions, respectively.

The suppression of the URCA rates due to superfluidity was calculated by using the results of Itoh (1971), Itoh and Tsuneto (1972), Wolf (1966) and Bahcall and Wolf (1965a). Itoh and Tsuneto (1972) also considered the URCA rates in subnuclear regions, and they were taken into account in our models. The recent work by Tsuruta et al. (1973) was used to take account of the URCA rates in the outermost layers (where $\varrho<\varrho_{1}$ ). These effects for heavy ions are generally negligible for heavier stars, but they become important for lighter stars.

The solid curve in Figure 7 shows the final, more realistic cooling curve for Model (II) which was obtained in the manner described above. To compare this result with the simplified version of the previous section, the dashed curves in Figure 7 show the two extreme cases, Case (S) (exaggerated superfluidity) and Case (N) (no superfluidity), for the same model.

Figure 8 shows the final cooling curves for our models (I), (II) and (III). We see that the lightest star (III) is cooler than others in earlier stages $\left(t \lesssim 10^{5} \mathrm{yr}\right)$ but the trend is reversed when the star becomes older. Model (II) is the coldest star in the

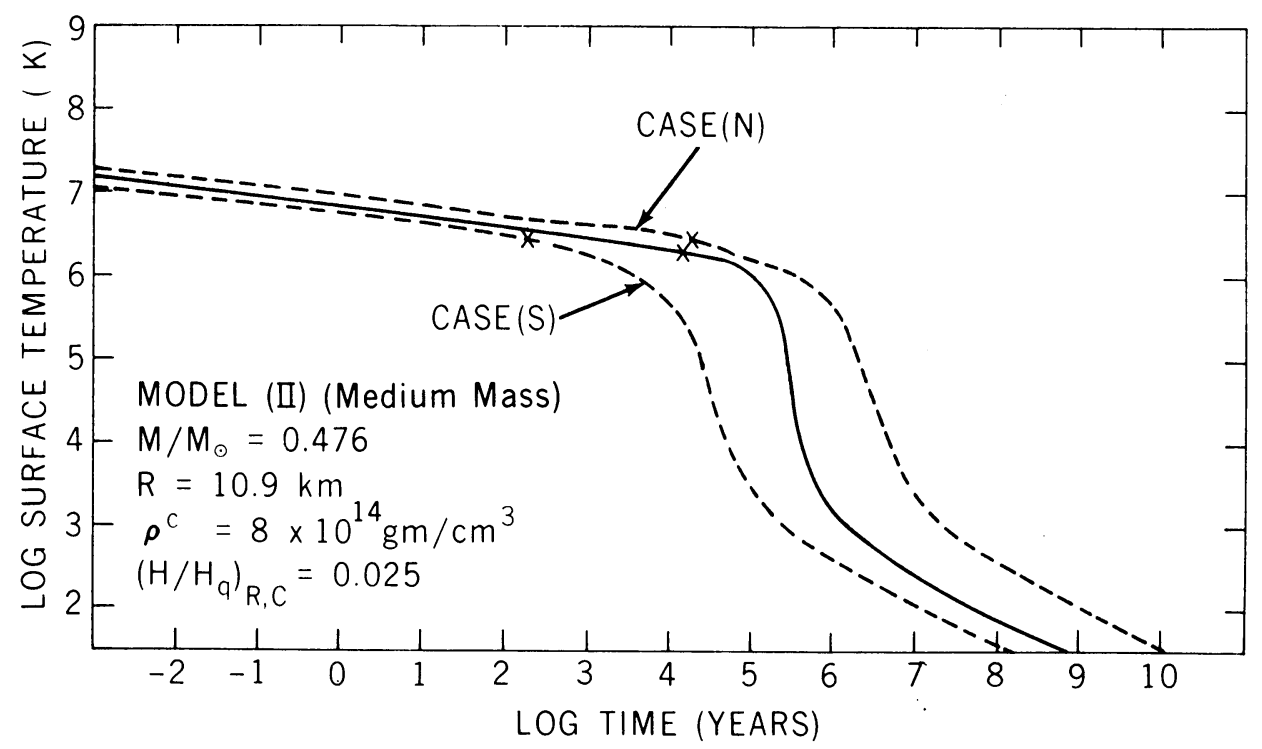

Fig. 7. Cooling curves (surface temperature vs time) for Model (II) of Section 3. The magnetic field strength is the same as in Figure 6. The dashed curves (S) and (N) are for maximum superfluidity and no superfluidity, respectively, as defined in Section 3.1. The solid curve is the final cooling curve obtained by the method described in Section 3.2. The crosses have the same meaning as in Figure 3. 


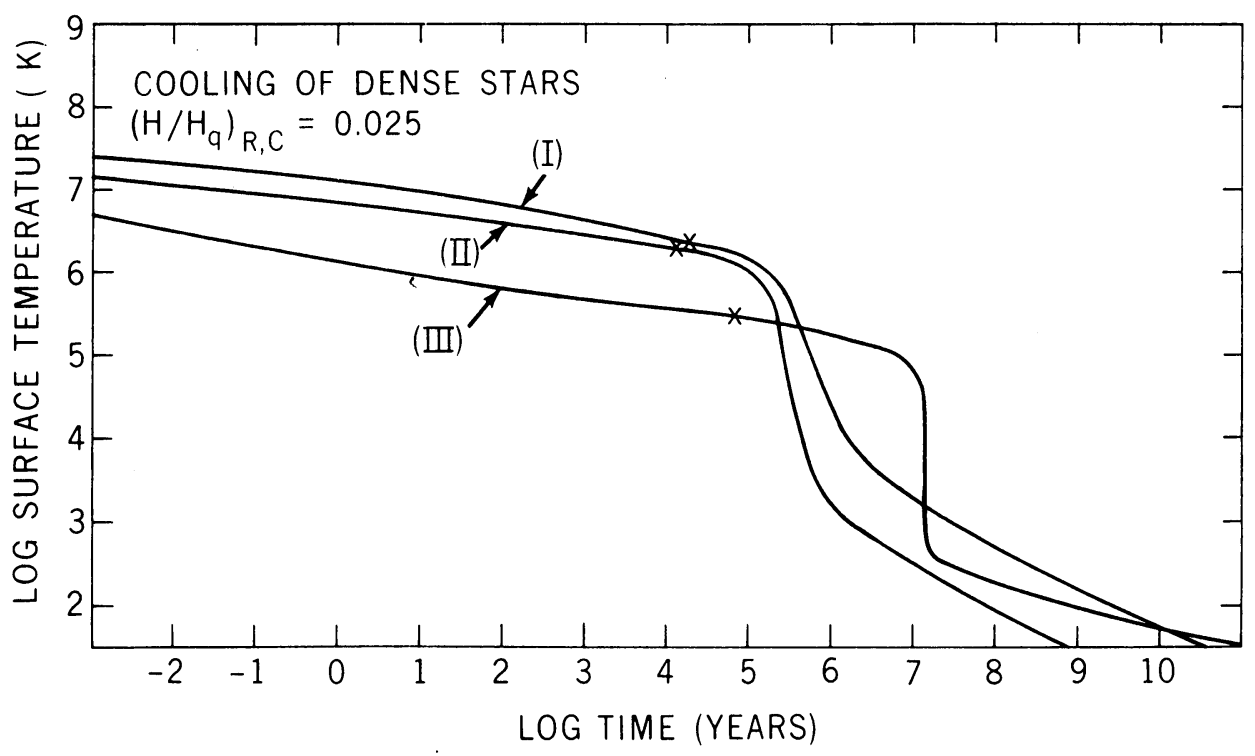

Fig. 8. Final cooling curves (surface temperature vs time) for the three models chosen in Section 3.2, with the same magnetic field strength as in Figure 6. The crosses have the same meaning as in Figure 3.

lower temperature regions where the effect of superfluidity becomes significant. At this point it may be pointed out that the major difference in cooling behavior comes from differences in density rather than mass differences. For instance, Model (II) of the present section and the $V_{\gamma}$ (II) model of the previous section, due to their similar densities, have similar cooling behaviors even though the latter is more than twice as massive as the former. Among realistic dense star models developed by other authors, our models chosen here are closest to those constructed by Baym et al. (1971b). (In the medium mass region our models are also similar to those constructed by Ikeuchi et al., 1971.)

Here we wish to emphasize the importance of heavy ions for lighter stars. A sudden drop of temperature takes place at the age of about $10^{7} \mathrm{yr}$ for Model (III) in Figure 8 (where heavy ion energies are included). When $U_{\text {ion }}^{N}$ is neglected in (2), this sudden cooling occurs near $10^{6}$ yr (for the same model).

At the age of a few million years, which is regarded as the average age of radio pulsars (e.g., Gunn and Ostriker, 1970), the stars should be very cold. This conclusion is relatively independent of the particular models used. This is because the transparency due to strong magnetic fields, which is the main cause for the sudden cooling, is achieved by this time. For those stars the surface temperatures are from several hundred to several thousand degrees. However, the surface may be kept at somewhat above $10^{5} \mathrm{~K}$ if we take into account the heating by accretion of interstellar matter (Ramaty, private communication). Thus, the conclusion reached in our previous work (Tsuruta et al., 1972) is still valid. Thermal ionization of atoms in the atmosphere will be negligible and only residual rotational energy may be responsible for these 
older pulsars. This may then explain the lack of observed pulsars with periods greater than a few seconds (Ruderman, 1971). These cold, dense stars seem to have a peculiar property, that we can see through to the center when we look down from the two magnetic poles. It may be noted that the above conclusion was reached by temporarily disregarding Model (III). This is because neutron stars with mass less than about $0.2 M_{\odot}$ may not be formed (Bethe, 1972; Cohen and Borner, 1972). (This lightest model was chosen to dramatize the density dependence of cooling behavior.) The cooling behavior of a neutron star of about $0.2 M_{\odot}$ is closer to our Model (II) than Model (III).

In Table II surface temperatures and internal temperatures of the three models are shown at a few interesting points during their cooling history. The first two points correspond to the approximate age of the Crab pulsar and Vela pulsar, respectively. There it seems likely that the star is hot enough so that thermal effects can be important. Earlier in this symposium it was reported that the pulsar glitch information allows some estimates of the mass of these younger pulsars. The mass of the Crab pulsar as estimated by Pines (in this volume) from the crustquake theory is about $0.5 M_{\odot}$, while it is greater than $1 M_{\odot}$ according to 'accretion' theory (Borner and

TABLE II

Surface temperatures $T_{\mathrm{e}}$ and internal temperatures $T_{1}$ for the models described in Table I, at three interesting points during the cooling history

\begin{tabular}{|c|c|c|c|c|c|}
\hline & \multicolumn{3}{|c|}{ Models } & \multirow{2}{*}{$\begin{array}{l}\text { Age } \\
\text { (years) }\end{array}$} & \multirow[t]{2}{*}{ Comment } \\
\hline & (I) & (II) & (III) & & \\
\hline $\log T_{\mathrm{e}}(\mathrm{K})$ & $\begin{array}{l}6.62 \\
6.40 \\
4.38\end{array}$ & $\begin{array}{l}6.45 \\
6.30 \\
3.21\end{array}$ & $\begin{array}{l}5.68 \\
5.56 \\
5.21\end{array}$ & $\begin{array}{l}10^{3} \\
10^{4} \\
10^{6}\end{array}$ & $\begin{array}{l}\sim \text { Crab } \\
\sim \text { Vela }\end{array}$ \\
\hline $\log T_{\mathrm{i}}(\mathrm{K})$ & $\begin{array}{l}8.62 \\
8.36 \\
4.58\end{array}$ & $\begin{array}{l}8.74 \\
8.54 \\
3.26\end{array}$ & $\begin{array}{l}8.54 \\
8.38 \\
7.92\end{array}$ & $\begin{array}{l}10^{3} \\
10^{4} \\
10^{6}\end{array}$ & $\begin{array}{l}\sim \text { Crab } \\
\sim \text { Vela }\end{array}$ \\
\hline
\end{tabular}

Cohen, 1972). If the core-quake theory applies, the Vela pulsar should be a heavy star (Shaham, 1972). Therefore, we may expect to be able to detect X-rays from these pulsars. It is already known that the Crab Nebula has an X-ray pulsar, though it is not of blackbody radiation. As of now I am not aware of any conclusive report about the detection of pulsed X-rays from the Vela pulsar. However, caution may be advised for these young pulsars because nucleon superfluidity, on which the above theories are based, may not yet be significant at their high temperatures. For instance, the Crab pulsar seems to too hot for the effect of the ${ }^{3} P_{2}$ superfluidity (see Table II). On the other hand, cooling will be much faster if pions are present (Bahcall and Wolf, 1965b; Tsuruta, 1972). It was already pointed out that pions may be present in high density regions (Sawyer, 1972; Scalapino, 1972). Even though we do not know yet 
exactly at what density pions appear, it is possible that they are abundant for heavy hadron stars.

At this point we wish to consider the possible heating effect of crust-core rotation slippage. If neutron star interiors are superfluid, then frictional heat may be dissipated as its rotation is slowed. This process may keep the star hotter for longer periods. [Such a possibility was first suggested by Cameron (1970).] Greenstein (1971) estimated this effect and concluded that it can be significant enough so that thermal X-ray emission from certain pulsars may be detectable. The comparison shows that both for the Crab pulsar and Vela pulsar the surface temperatures in his results and those obtained in this section (Table II) are comparable if they are medium weight to heavy stars (the Models (II) and (I)). Greenstein suggests a few more older pulsars as other possible candidates for detection of X-rays due to this heating effect. Their surface temperatures estimated by Greenstein are in the vicinity of $(2 \sim 3) \times 10^{5} \mathrm{~K}$. At the ages which are expected for these pulsars Model (III) is at comparable temperatures but our Models (II) and (I) are colder (with their surface temperatures at $10^{3} \sim 2 \times 10^{5} \mathrm{~K}$ ). (We may comment that the magnitude of the frictional effects depends on the strength of the coupling between the charged and the superfluid components of the stars and that quantitative estimates are not yet available.)

\subsection{COOLING OF WHITE DWARFS}

Before closing we wish to show typical cooling curves for white dwarfs. The properties of the particular model chosen here are listed in Table III. This is the same model as the one chosen in Tsuruta and Cameron (1970) and further details are found in that paper. A rather massive white dwarf star was chosen so that it can possess an URCA shell. However, other neutrino processes (Beaudet et al., 1967; Hansen, 1968) are also included here. The cooling curves thus obtained are shown in Figure 9. A simple ideal gas was assumed for the straight line, while the other curve includes the effect of crystallization.

TABLE III

Properties of the white dwarf model chosen in Section 3.3

\begin{tabular}{|c|c|c|}
\hline & \multicolumn{2}{|c|}{$\begin{array}{l}\text { Mass }=1.373 M_{\odot} \\
\text { Central density }=10^{9.5} \mathrm{~g} \mathrm{~cm}^{-3} \\
\text { Radius }=1810 \mathrm{~km} \\
\text { Radius of URCA shell }=377 \mathrm{~km} \\
\text { URCA nuclear pair }{ }^{23} \mathrm{Na} \text { and }{ }^{23} \mathrm{Ne} \\
\text { Core of } 1 M_{\odot} \text { of carbon-burning products } \\
\text { Envelope of }{ }^{12} \mathrm{C}\end{array}$} \\
\hline \multicolumn{3}{|c|}{ Composition of Core } \\
\hline Mass number & Mass fraction & $\begin{array}{l}\text { Nuclei and electron capture thresholds } \\
(\mathrm{MeV})\end{array}$ \\
\hline 16 & 0.01 & ${ }^{16} \mathrm{O}(10.4)^{16} \mathrm{C}$ \\
\hline 20 & 0.41 & ${ }^{20} \mathrm{Ne}(7.03)^{20} \mathrm{O}(21.22)^{20} \mathrm{C}$ \\
\hline 23 & 0.06 & ${ }^{23} \mathrm{Na}(4.4)^{23} \mathrm{Ne}(11.15)^{23} \mathrm{~F}(13.87)^{23} \mathrm{O}$ \\
\hline 24 & 0.52 & ${ }^{24} \mathrm{Mg}(5.52)^{24} \mathrm{Ne}(15.91)^{24} \mathrm{O}$ \\
\hline
\end{tabular}




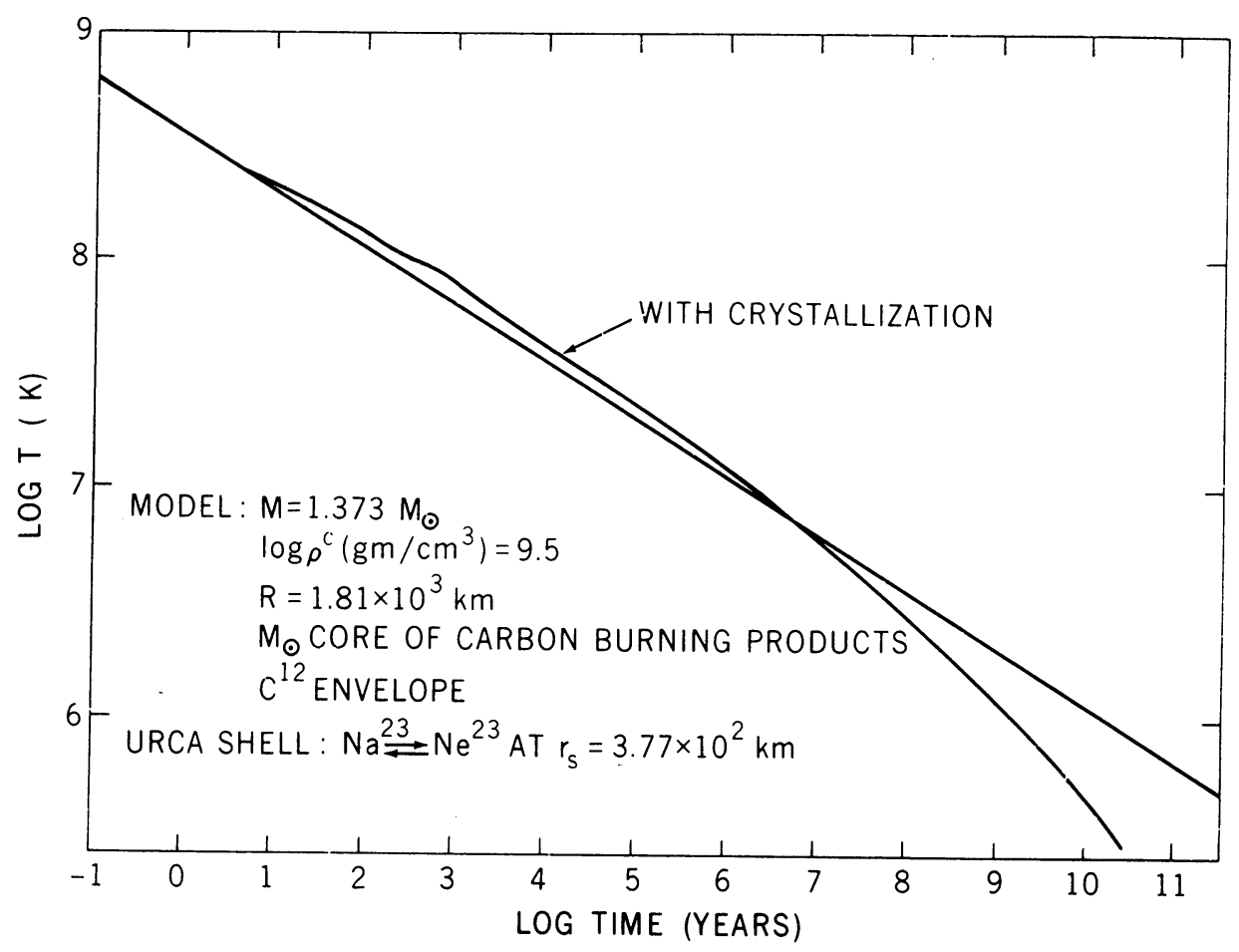

Fig. 9. Cooling curves for the white dwarf model chosen in Section 3.3. The straight line is for an ideal gas. In the other curve, the effect of crystallization is included.

\section{Acknowledgement}

I wish to thank the many persons mentioned in this paper and other participants of this symposium for valuable suggestions and discussions. The last part of this paper was completed during my visit at the Aspen Center for Physics.

\section{References}

Bahcall, J. N. and Wolf, R. A.: 1965a, Phys. Rev. 140, B1452.

Bahcall, J. N. and Wolf, R. A.: 1965b, Phys. Rev. Letters 14, 343.

Baym, G., Bethe, H. A., and Pethick, C. J.: 1971a, Nucl. Phys. A175, 225.

Baym, G., Pethick, C. J., and Sutherland, P.: 1971b, Astrophys. J. 170, 299.

Beaudet, G., Petrosian, V., and Salpeter, E. E.: 1967, Astrophys. J. 150, 979.

Bethe, H. A.: 1972 (private communication).

Borner, G. and Cohen, J. M.: 1972, Pulsar Speed-ups (preprint).

Cameron, A. G. W.: 1970 (private communication).

Canuto, V.: 1970, Astrophys. J. 160, L153.

Canuto, V. and Chiu, H.-Y.: 1969, Phys. Rev. 188, 2446.

Canuto, V., Lodenquai, J., and Ruderman, M.: 1971, Phys. Rev. D3, 2303.

Cohen, J. M. and Borner, G.: 1972 (private communication).

Ginzburg, V. L.: 1971, Physica 55, 207.

Gold, T.: 1968, Nature 218, 731.

Greenstein, G.: 1971, Nature Phys. Sci. 232, 117. 
Gunn, J. E. and Ostriker, J. P.: 1970, Astrophys. J. 160, 979.

Hansen, C. J.: 1968, Astrophys. Space Sci. 1, 499.

Hartle, J. B. and Thorne, K. S.: 1968, Astrophys. J. 153, 807.

Ikeuchi, S., Nagata, S., Mizutani, T., and Nakazawa, K.: 1971, Prog. Theor. Phys. 46, 95.

Itoh, N.: 1971 (private communication).

Itoh, N. and Tsuneto, T.: 1972, Prog. Theor. Phys. Kyoto 48, 1849.

Landau, L. D. and Lifshitz, E. M.: 1958, Statistical Physics, Addison-Wesley Publ. Co., Mass.

Lodenquai, J., Canuto, V., Ruderman, M., and Tsuruta, S.: 1974 (to be published).

Mestel, L. and Ruderman, M.: 1967, Monthly Notices Roy. Astron. Soc. 136, 27.

Moszkowski, S. A.: 1972, paper presented at APS Meeting, April, Washington D. C. (Bulletin of APS 17, 502).

Pandharipande, V. R.: 1971, Nucl. Phys. A178, 123.

Ravenhall, D. G., Bennett, C. D., and Pethick, C. J.: 1972, Nuclear Surface Energy and Neutron Star Matter (preprint), (1972, Phys. Rev. Letters 28, 978).

Ruderman, M.: 1971, Phys. Rev. Letters 27, 1306.

Salpeter, E. E.: 1961, Astrophys. J. 134, 669.

Sawyer, R. F.: 1972, Phys. Rev. Letters 29, 382.

Scalapino, D. J.: 1972, Phys. Rev. Letters 29, 386.

Schatzman, E.: 1958, White Dwarfs, North-Holland Publ. Co, Amsterdam.

Shaham, J.: 1972 (private communication).

Tamagaki, R.: 1968, Prog. Theor. Phys. Kyoto 39, 91.

Tamagaki, R.: 1972 (private communication).

Takatsuka, T.: 1972, Energy. Gap in Neutron-Star Matter (preprint) NEAP-11, May, Kyoto University, (1972, Prog. Theor. Phys. Kyoto 48, 1517).

Tsuruta, S.: 1964, Thesis, Columbia University.

Tsuruta, S.: 1974 (to be published).

Tsuruta, S. and Cameron, A. G. W.: 1966a, Can. J. Phys. 44, 1863.

Tsuruta, S. and Cameron, A. G. W.: 1966b, Can. J. Phys. 44, 1895.

Tsuruta, S. and Cameron, A. G. W.: 1970, Astrophys. Space Sci. 7, 374.

Tsuruta, S., Canuto, V., Lodenquai, J., and Ruderman, M.: 1972, Astrophys. J. 176, 739.

Tsuruta, S., Truran, J. W., and Cameron, A. G. W.: 1973, Bulletin of APS 18, 541.

Van Horn, H. M.: 1968, Astrophys. J. 151, 227.

Wheeler, J. A.: 1966, Ann. Rev. Astron. Astrophys. 4, 393.

Wolf, R. A. 1966, Astrophys. J. 145, 834.

\section{DISCUSSION}

Greenstein: I notice that some of your temperatures derived for the crab pulsar can get quite high - on the order of $10^{9} \mathrm{~K}$ in the interior. This is of the same order of the superfluid energy gap in some regions. If so, then some of the superfluid in this pulsar could contain lots of normal component. Situations of this sort have never been considered by neutron star superfluidists.

Van Horn: Your curves of $\log T_{\mathrm{e}}$ vs $\log t$ show a rapid decrease of the temperature at an age of $10^{4}-10^{6}$ yr. Could you explain the reason for this?

Tsuruta: The effect is due to the decrease of opacity in the presence of magnetic fields.

Itoh: I would like to comment on the opacity of the region where a neutron liquid, a proton liquid, and an electron liquid coexist. Recently, Pethick, Flowers and I have calculated the thermal conductivity of this region and find that electrons and neutrons contribute comparably to the thermal conductivity when nucleons form normal Fermi liquids. 